\title{
Comparative Studies of Poly(Dimethyl Siloxanes) Using Automated GPC-MALDI- TOF MS and On-Line GPC-ESI-TOF MS
}

\author{
X. Michael Liu, E. Peter Maziarz, David J. Heiler, and George L. Grobe \\ Research, Development, and Engineering, Bausch and Lomb, Rochester, New York, USA
}

In this study we compare on-line gel permeation chromatography (GPC) electrospray ionization (ESI) time-of-flight (TOF) mass spectrometry (MS) to automated GPC matrix assisted laser desorption ionization (MALDI) TOF MS for poly (dimethylsiloxane) (PDMS) analysis. Average mass values for a hydroxyl-terminated PDMS (OH-PDMS) sample were obtained and compared to traditional GPC that was calibrated with narrow polystyrene standards, by direct ESI and MALDI MS analysis, by a summation of mass spectra of all GPC fractions, and also by the recalibration method determined by both mass spectrometric methods. Quantitatively, the difference noted here between these hyphenated techniques is that GPC-ESI-TOF MS effectively reports the low-mass oligomers and underestimates the high-mass oligomers, while GPC-MALDI-TOF MS effectively reports the high-mass oligomers and underestimates the low-mass oligomers. In the GPC-ESI-TOF MS experiments, ion current suppression was observed in the high molecular weight region. The suppression effect was confirmed by repeatable sample runs and by injecting different PDMS samples. Higher chromatographic resolution was observed for GPC-ESI-TOF MS compared to GPC-MALDITOF MS. In fact, truly mono-disperse oligomers were observed in the low molecular weight range from GPC-ESI MS experiments. (J Am Soc Mass Spectrom 2003, 14, 195-202) (c) 2003 American Society for Mass Spectrometry

$\mathrm{T}$ The advent of soft ionization techniques such as matrix-assisted laser desorption ionization (MALDI) and electrospray ionization (ESI) have provided chemists the ability to evaluate polymer samples with several different mass analyzers. Specifically for polysiloxane materials the information rich data obtained from such analysis has been used qualitatively for repeat unit and end group determination [1-7] and quantitatively for relative concentration $[8,9]$ and average mass determination $[10,11]$.

Several reviews appear in the literature that highlight many of the applications of polymer mass spectrometry analysis [12-15]. The general consensus for determining average mass values is that the accuracy of such measurements is limited to polymer samples having a relatively narrow polydispersity (PD) index of less than 1.2 [16-20]. Mass discrimination occurs with more broadly dispersed polymer samples such that the high molecular weight oligomers within the molecular weight distribution (MWD) are under-represented or not observed at all. Several reports have been written that hypothesize of the root cause for these discrimination effects [21-28].

Published online January 24, 2003

Address reprint requests to Dr. X. M. Liu, Research, Development, and Engineering, Bausch and Lomb, 1400 North Goodman Street, P.O. Box 30450, Rochester, NY 14603-0450, USA. E-mail: x.michael.liu@bausch.com
One strategy to overcome these mass discrimination effects is to incorporate gel permeation chromatography (GPC) prior to mass spectrometry. GPC separates molecules based on their hydrodynamic volumes where large molecules elute from the column before the smaller molecules $[29,30]$. With the GPC-MS combination the complexity of a broad MWD is minimized by independently evaluating nearly mono-disperse chromatographic fractions. This mass fraction data can be used to recalibrate the GPC trace to obtain absolute average mass values. Additionally, the MWD can be reconstructed by summing the mass fraction data from which average mass values can be computed. Several research groups have illustrated the powerful combination of GPC with MALDI and ESI MS for polymer analysis. In the most traditional sense, GPC can be incorporated by manually collecting GPC fractions in a number of vials for subsequent analysis by ESI $[23,31]$ or MALDI MS $[5,6,10,11,23,32-36]$. This approach is relatively time consuming and very tedious since each collected GPC mass fraction requires sample preparation prior to MS analysis. Alternatively, GPC can be combined with ESI or MALDI MS in a more automated approach. For example, the solution infusion characteristics of GPC and ESI allow these techniques to be combined on-line [37-40]. Fei and Murray [41] have reported on-line GPC coupling to a MALDI source. However, to date most GPC-MALDI analysis employs 
deposition of the GPC effluent onto a MALDI sample target followed by mass analysis [42-49]. This method is not considered to be true on-line hyphenation, when compared to GPC-ESI MS, since the deposition of GPC effluent and subsequent mass analysis occurs in two discrete time events. Rather, within this context it is considered to be automated GPC-MALDI MS.

In this study we compare on-line GPC-ESI MS to automated GPC-MALDI MS for polymer analysis and as such it seems proper to use the polymer's average mass values obtained by traditional GPC analysis. Although traditional GPC provides polystyrene-equivalent average molecular weights, it remains useful as a benchmark in this comparative study.

\section{Experimental}

\section{Poly(Dimethyl Siloxane) Materials and Chemicals}

The three PDMS polymers with different end groups that include OH-PDMS, methyl-terminated (MEPDMS), and $\alpha, \omega$-bis(4-hydroxylbutyl)-terminated (HBPDMS) were synthesized in-house from previously described procedures [48]. Sodium nitrate and dithranol were obtained from Aldrich (Milwaukee, WI). HPLC grade tetrahydrofuran (THF) and isopropanol (IPA) were all purchased from Fisher Scientific (Fairlawn, NJ).

\section{Gel Permeation Chromatography}

The solvent delivery system used a Waters Alliance 2690 Separation Module (Waters Corporation, Milford, MA). All samples were prepared in THF at a concentration of $0.12 \%$ (wt/vol). The separation was performed on a set of two PL-gel mixed-E columns that were purchased from Polymer Laboratories, Amherst, MA. The dimension of the columns were $300 \mathrm{~mm} \times 7.8$ $\mathrm{mm}$ and the particle size of the packing materials was 3 $\mu \mathrm{m}$. Fifty $\mathrm{mL}$ of the sample solution were injected into the GPC system. HPLC grade tetrahydrofuran was used as the mobile phase that was flowed at 1.0 $\mathrm{mL} / \mathrm{min}$. The column temperature was set at $35^{\circ} \mathrm{C}$. A series of narrow polystyrene molecular weight standards (Polymer Laboratories) with molecular weight ranging from 30,000 to $500 \mathrm{Da}$ were used to calibrate the GPC column set. Oligomeric separation of the $500 \mathrm{Da}$ polystyrene standard was achieved because of high chromatographic resolution. A Waters 2410 refractive index detector was used to monitor the GPC eluent. The internal temperature of the refractive index detector was set at $35^{\circ} \mathrm{C}$.

\section{Thermal Spray Device (GPC-MALDI-TOF MS Interface)}

The automated GPC-MALDI MS employed an LCTransform Series 500 interface (Lab Connections, Inc., Northborough, MA). This system was modified for a matrix co-deposition mechanism with GPC effluent and has been previously described [43]. Dithranol was used as the matrix and was prepared as $15 \mathrm{mg} / \mathrm{mL}$ in HPLC grade THF with the addition of $2 \%(\mathrm{vol} / \mathrm{vol})$ saturated sodium nitrate in THF. The matrix solution flow rate was $0.2 \mathrm{~mL} / \mathrm{min}$ and directed towards a Valco (Houston, TX) tee connector where mixing with GPC effluent occurred prior to deposition on the MALDI sample target. The nozzle temperature was set at $193^{\circ} \mathrm{C}$ and the nitrogen sheath gas was adjusted to a pressure of $25 \mathrm{psi}$. While evaporating most of the solvent, the uniform micro-cocrystals between sample molecules and matrix were formed because of the well-controlled experimental conditions. After the GPC experiment, the MALDI target was subjected to MALDI-TOF MS analysis.

\section{MALDI-TOF Mass Spectrometry}

The MALDI-TOF MS data was obtained with an Applied Biosystems Voyager DE-STR TOF (Framingham, MA) mass spectrometer, operating in the reflector mode. Ions were formed by laser desorption at $337 \mathrm{~nm}$ $\left(\mathrm{N}_{2}\right.$ laser, $3 \mathrm{~ns}$ pulse width, $10^{6} \mathrm{~W} / \mathrm{cm}^{2}, 100 \mu \mathrm{m}$ diameter spot), accelerated to $25 \mathrm{kV}$ and detected as positive ions. During the ionization process a delay time of 100 to $175 \mathrm{~ns}$ was applied before acceleration. Additionally, the grid and guide wire voltages were set at 75.0 and $0.030 \%$ of the applied acceleration voltage, respectively, to focus the beam of ions. Typically, 256 laser shots were averaged for each spectrum.

\section{GPC-ESI-TOF Mass Spectrometry}

The solvent delivery system for on-line GPC ESI-TOF MS experiments used an Agilent (Palo Alto, CA) 1100 HPLC system. The GPC column set and all other GPC experimental conditions used were the same as described in the gel permeation chromatography section. The ESI-TOF MS data was obtained with an Applied Biosystems Mariner mass spectrometer equipped with a Turbo electrospray ion source (Applied Biosystems). The experimental set-up is somewhat similar to that described by Simonsick and Prokai [38]. The main difference between the two designs is the location where charge agent and GPC effluent mixing occurs. Specifically, in their set up the charge agent solution was coaxially sprayed with GPC effluent and thus mixing occurred at the end of the spray needle. For the experiment here, the charge agent solution and GPC effluent were mixed in a Valco Cross connector before the ESI source. The charge agent solution consisted of isopropanol (IPA) with $2 \%(\mathrm{vol} / \mathrm{vol})$ of saturated sodium nitrate solution in IPA. A built-in syringe pump at a flow rate of $10 \mu \mathrm{l} / \mathrm{min}$ delivered the charge agent solution. The split ratio to the waste and to the ESI-MS was solely controlled and adjusted by the internal diameter and length of the waste line tubing. The total flow rate directed to the ESI MS instrument was determined to be approximately $150 \mu \mathrm{l} / \mathrm{min}$. The spray tip 
Table 1. Average molecular mass values and molecular weight distributions of the OH PDMS obtained from different analytical techniques

\begin{tabular}{lrrrr}
\hline MW & & & & \\
Analytical techniques & $\mathrm{M}_{\mathrm{n}}$ & $\mathrm{M}_{\mathrm{w}}$ & $\mathrm{M}_{\mathrm{z}}$ & $\begin{array}{c}\text { Polydispersity } \\
\left(\mathrm{M}_{\mathrm{w}} / \mathrm{M}_{\mathrm{n}}\right)\end{array}$ \\
\hline \hline Conventional GPC & 796 & 1112 & 2221 & 1.40 \\
MALDI-TOF MS & 751 & 844 & 945 & 1.12 \\
ESI-TOF MS & 635 & 672 & 3910 & 1.06 \\
GPC-MALDI-TOF MS by spectra-summing & 1830 & 2909 & 3311 & 1.59 \\
GPC-MALDI-TOF MS by recalibration & 1063 & 1400 & 933 & 1.32 \\
GPC-ESI-TOF MS by spectra-summing & 623 & 731 & 1782 & 1.17 \\
GPC-ESI-TOF MS by recalibration & 641 & 867 & & \\
\hline
\end{tabular}

and nozzle potentials were set at 5000 and 100 volts, respectively. Positive ion mode was used for all ESI MS measurements.

\section{Data Analysis}

The molecular weights and molecular weight distributions of a polymer were calculated by the following formulae:

$$
\begin{aligned}
& \mathrm{M}_{\mathrm{n}}=\Sigma\left(\mathrm{N}_{\mathrm{i}} * \mathrm{M}_{\mathrm{i}}\right) / \Sigma \mathrm{M}_{\mathrm{i}} \\
& \mathrm{M}_{\mathrm{w}}=\Sigma\left(\mathrm{N}_{\mathrm{i}} * \mathrm{M}_{\mathrm{i}}^{2}\right) / \Sigma\left(\mathrm{N}_{\mathrm{i}} * \mathrm{M}_{\mathrm{i}}\right) \\
& \mathrm{M}_{\mathrm{z}}=\Sigma\left(\mathrm{N}_{\mathrm{i}} * \mathrm{M}_{\mathrm{i}}^{3}\right) / \Sigma\left(\mathrm{N}_{\mathrm{i}} * \mathrm{M}_{\mathrm{i}}^{2}\right) \\
& \mathrm{PD}=\mathrm{M}_{\mathrm{w}} / \mathrm{M}_{\mathrm{n}}
\end{aligned}
$$

where $M_{n}, M_{w}$, and $M_{z}$ represent number-average, weight-average, and z-average molecular mass, respectively and where $N_{i}$ is the number of polymer molecules at molecular mass $\mathrm{M}_{\mathrm{i}}$. PD is the polydispersity index or molecular weight distribution. These average mass values were determined from GRAMS 32 (Galactic Industries, Salem, NH) and Millennium 32 software (Waters Corporation).

\section{Results and Discussion}

\section{Comparison of Average Mass Values by On-Line GPC-ESI and Automated GPC-MALDI-TOF MS Analysis}

Average mass values for a relatively low molecular weight hydroxyl terminated PDMS (OH-PDMS) sample were obtained from traditional GPC that was calibrated with narrow polystyrene standards from direct ESI and MALDI MS analysis and from a summation of mass spectra of OH PDMS fractions also determined by both mass spectrometric methods. The determined average mass values are summarized in Table 1. From traditional GPC analysis the PD of the OH-PDMS polymer was determined to be 1.40 , indicating a relatively broad molecular weight distribution.

It is well documented that direct MS analysis under- estimates the MWD of polydispersed polymers. From our experiments the PD of the OH-PDMS sample evaluated by direct ESI and MALDI MS differs from traditional GPC by 24.3 and $20.0 \%$, respectively. To approximate where in the MWD the MS method underestimates the oligomer population one can compare the first, second, and third order average mass values $\left(M_{n}, M_{w}\right.$, and $M_{z}$, respectively) which are increasingly dependant on the higher mass oligomers within the MWD. For both direct ESI and MALDI MS the determined $\mathrm{M}_{\mathrm{z}}$ average mass differs from GPC by 67.5 and $57.5 \%$ respectively. This indicates that direct ESI-TOF and MALDI-TOF MS analysis of this polymer significantly underestimates the higher mass oligomers within the molecular weight distribution.

Figure $1 a$ and $b$ illustrate the differential refractive index trace from GPC analysis and the total ion chromatogram (TIC) from on-line GPC-ESI MS analysis, respectively. The average masses in Table 1 for GPC-ESI MS were obtained by summing all the mass spectra from 11 to $16 \mathrm{~min}$ on the TIC (Figure 1a). Based on traditional GPC analysis this time frame should include the entire MWD for the sample. A PD of 1.17 was determined using the spectra-summing technique. This PD value is $16.4 \%$ lower than the traditional GPC analysis and is only marginally better than that obtained from direct ESI-TOF MS analysis. From the

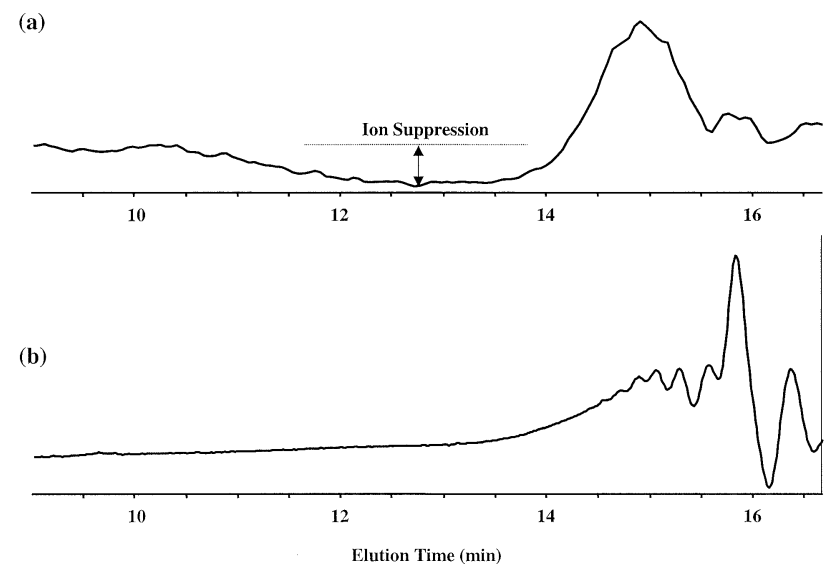

Figure 1. Total ion chromatogram (a) and dRI chromatogram (b) of the OH-PDMS sample in on-line GPC-ESI-TOF MS experiments. 
relatively large difference of $58.0 \%$ in calculated $\mathrm{M}_{\mathrm{z}}$ average mass compared to conventional GPC it seems that the GPC-ESI MS experiment still considerably underestimates the high molecular weight oligomers within the MWD for this polymer. The average masses in Table 1 for GPC-MALDI MS were obtained by summing spectra within the same time limits (11 to 16 $\mathrm{min}$ ) as for the GPC-ESI-TOF MS experiment. A PD value of 1.59 was calculated from the GPC-MALDI-TOF MS data. Although this value has a relatively low difference of $13.6 \%$ compared to traditional GPC, the average mass values of $M_{n}, M_{w}$, and $M_{z}$ are significantly higher than that calculated by traditional GPC. This indicates that GPC-MALDI-TOF MS effectively reports the high-mass polymers within the MWD, however the low-mass polymers are under-reported and it is proposed to be mainly due to the mass cutoff at 550 Da for the elimination of matrix interferences. Another possibility for this mass bias is that the low mass PDMS polymers have higher volatility than that of high mass PDMS polymers as suggested in a study by Yan et al. [9]. Under vacuum conditions in MALDI-TOF MS experiments, some of the low mass oligomers go into the gas phase as a result of evaporation without getting ionized through the normal MALDI process. This proposal may in fact be valid for dried droplet sample preparations where it is common to have heterogeneous mixing between matrix and oligomers. However, in this study the sample preparation is performed with a thermal spray deposition process where relatively homogeneous microcrystalline matrix and sample molecules are generated. Here, sample molecules are homogeneously embedded in the matrix $[43,50]$ and thus differences in volatility between the low and high mass oligomers should not be crucial.

There are several reasons for the differences in reported average mass values noted between GPC-ESI and GPC-MALDI-TOF MS analysis for this OH-PDMS polymer. The most fundamental of these reasons relates to the different underlying principles of operation (between ESI and MALDI) that govern ion transformation of PDMS polymer molecules into the gas phase. We also believe that these differences can in part be due to the differences in versatility between GPC-ESI and GPCMALDI-TOF MS methods. GPC-MALDI is an off-line technique and as such the fractionated oligomers are isolated on the MALDI sample plate almost indefinitely. Even after several MALDI measurements, most of the fractionated polymer sample remains unconsumed. This makes it very convenient to optimize MALDI experimental parameters (grid voltage, delayed extraction etc.) so as to achieve optimal signal-to-noise $(\mathrm{S} / \mathrm{N})$ ratio of the oligomer peaks throughout the entire MWD. Also, with essentially unlimited analysis time it is possible to sum many scans for one particular GPC fraction. This can be especially beneficial for observing the less concentrated and less efficiently desorbed high mass polymers within the MWD.

With the inherent real time analysis of the on-line

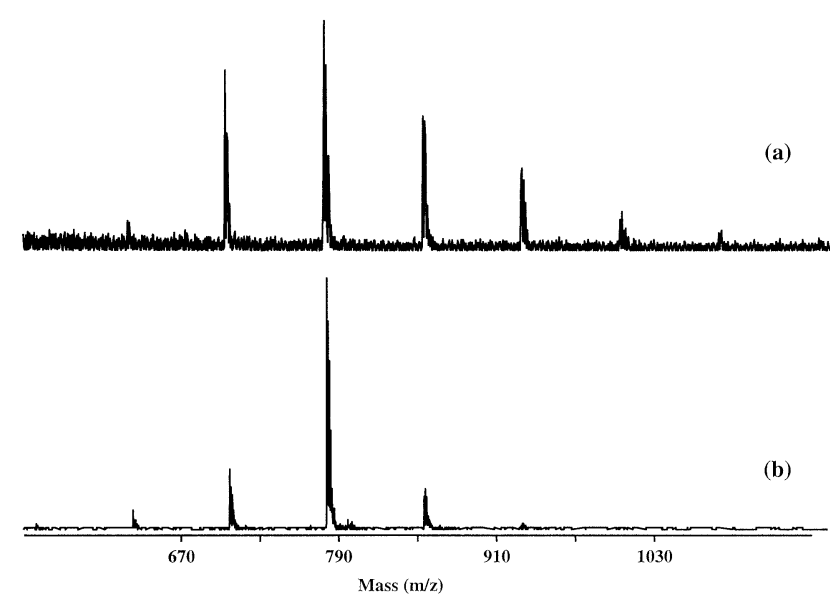

Figure 2. Typical mass spectra of PDMS samples at the same elution time from automatic GPC-MALDI-TOF MS experiment (a) and from on-line GPC-ESI-TOF MS experiment (b).

GPC-ESI-TOF MS there is very restricted amount of analysis time available and as such this limits the number of spectra that can be summed per sample analysis. The limited analysis time per mass spectrum makes it difficult to optimize ESI experimental conditions so that high mass polymers within the MWD can be observed. Also, it is unlikely that one constant optimal set of experimental conditions be applied for all the oligomers within a broad MWD. There are at least two analytical strategies that can be employed to compromise the experimental conditions for low and high mass polymers. First, ESI experimental parameters can be optimized from direct analysis of the non-fractionated polymer sample. However, this can be troublesome for optimization of the high mass polymers since these compounds in a polydisperse polymer sample are discriminated and perhaps not observed at all. Thus, optimization must occur on low to moderate mass polymers within the MWD, which according to the study here does not help to observe the high mass polymers within the MWD once GPC is applied. A second strategy would be to isolate a high mass fraction in a vial and optimize experimental parameters from direct analysis of this high mass fraction. However, this precludes the notion of high-throughput analysis of polymer samples for which we are attempting to develop a method.

\section{Evaluation of the Mass Spectra}

For both hyphenated techniques the same GPC column set was used. Figure 2 illustrates mass spectra from the same elution time analyzed by GPC-ESI and GPCMALDI-TOF MS methods. In both Figure $2 a$ and $b$ the decamer is the most intense oligomer observed. This confirms that the GPC in both hyphenated techniques have similar size exclusion properties. However, there are seven oligomers observed the GPC-MALDI experiment (Figure 2a) and five oligomers observed in the GPC-ESI experiment (Figure 2b). Moreover, in the GPC 

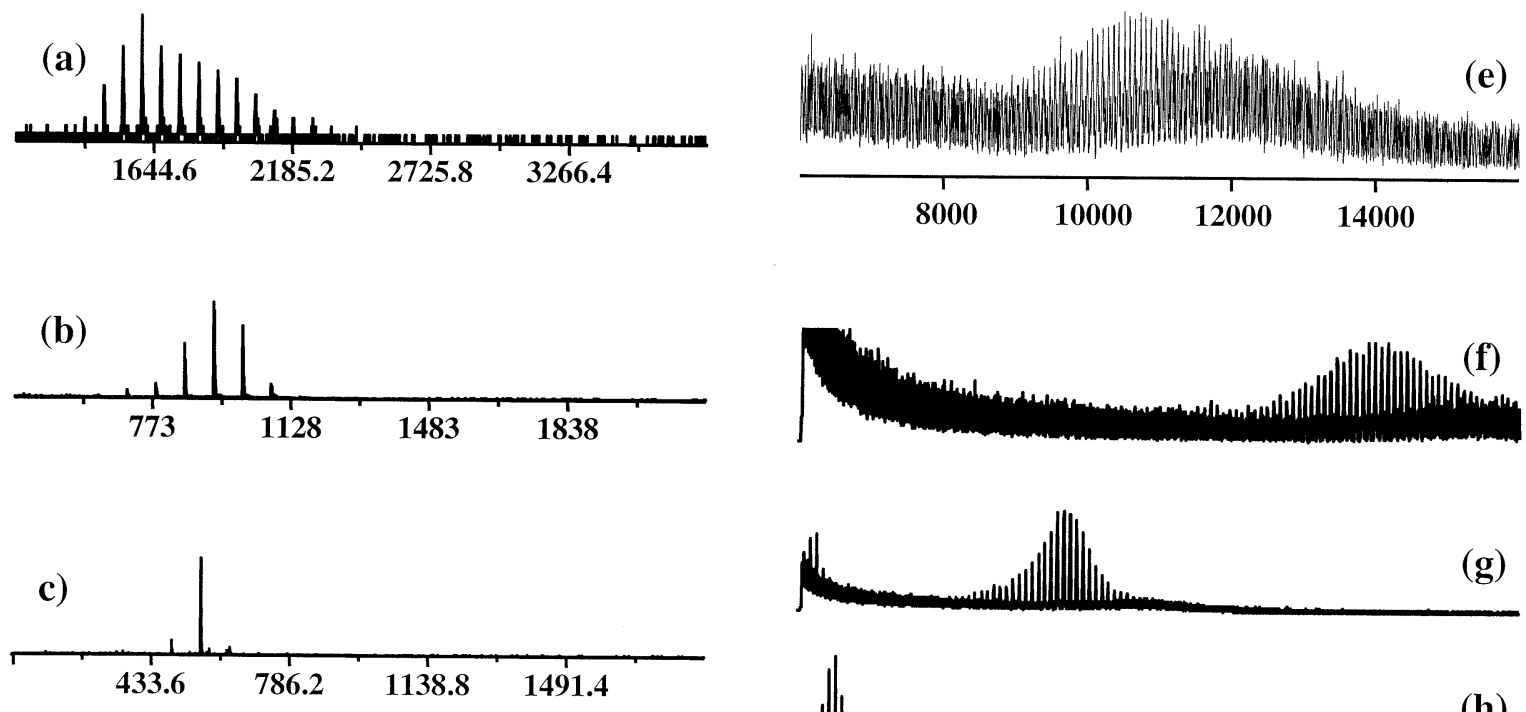

(g)
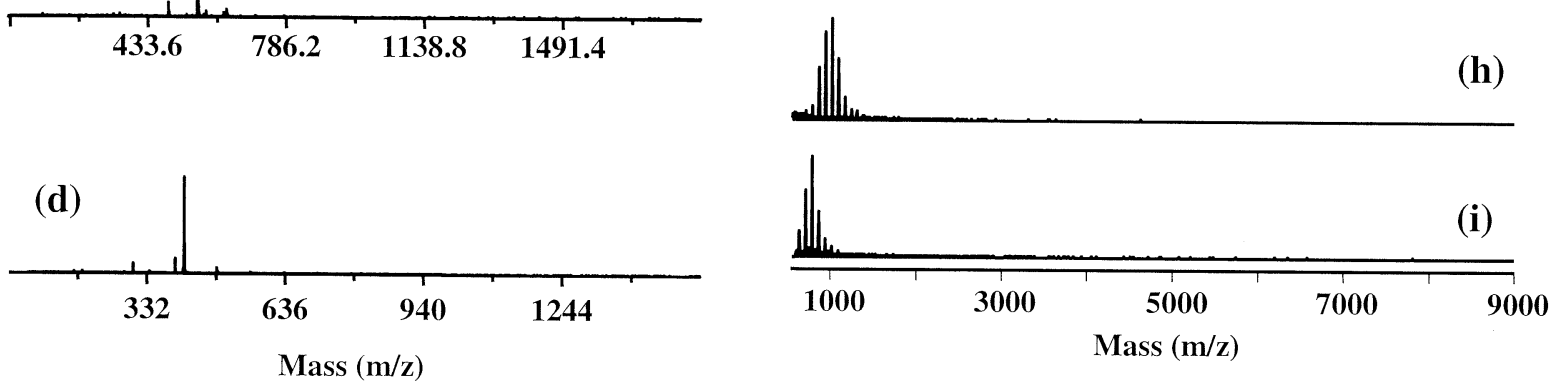

Figure 3. Overlaid mass spectra of the OH-PDMS obtained from both hyphenated techniques. Traces from (a) to (d) represent the mass spectra eluted sequentially at 13.5, 14.7, 15.0, and 15.3 min from on-line GPC-ESI-TOF MS experiments. Traces from (e) to (i) represent the mass spectra sequentially eluted at 10.5, 11.0, 12.4, 14.0, and $14.6 \mathrm{~min}$ from automatic GPC-MALDI-TOF MS experiments.

ESI experiment the ratio of intensities between the adjacent oligomers and the decamer is less than $25 \%$. Comparatively, this ratio is approximately $80 \%$ in the GPC-MALDI experiment. The lower chromatographic resolution in the GPC-MALDI experiment can be explained by the potential mixing of oligomers that can occur sometime during the thermal-sprayed deposition process.

Figure $3(\mathrm{a}-\mathrm{d})$ and $3(\mathrm{e}-\mathrm{i})$ illustrate mass spectra at different elution times for the GPC-ESI and GPCMALDI-TOF MS experiments, respectively. Very narrow polydispersed polymer fractions were obtained in each spectrum as a result of GPC fractionation. Performance of the GPC-ESI MS analysis was very efficient in the low molecular weight range $(<2000 \mathrm{Da})$. In fact Figure $3 c$ and $d$ indicate truly mono-disperse fractions as they contain single OH-PDMS oligomers in the low molecular weight range; namely the sodiated pentamer and heptamer, respectively. Observation of these single oligomers indicates the efficient GPC column set employed and confirms that the on-line GPC-ESI MS interface set-up has extremely low dead volume. Much lower ion intensities are observed in the higher mass region (>2000 Da) and the highest molecular mass observed is under $4000 \mathrm{Da}$. GPC-MALDI-TOF MS analysis of the same polymer resulted in mass spectra with excellent $\mathrm{S} / \mathrm{N}$ ratio in the higher mass region as illustrated in Figure $3(\mathrm{e}-\mathrm{g})$. The highest oligomeric mass observed was approximately 12,000 Da (Figure 3e). Overlapping matrix peaks compromised the quality of mass spectra below $600 \mathrm{Da}$ and no certainty could be given to the observation of oligomer peaks below 550 Da. The major difference between these techniques for the analysis of this OH-PDMS polymer sample is that GPC-ESI MS effectively reports the low-mass oligomers and underestimates the high-mass oligomers, while GPC-MALDI MS effectively reports the high-mass oligomers and underestimates the low-mass oligomers.

Regarding discrimination of the high mass polymers by GPC-ESI MS, an interesting observation can be noted in the TIC (Figure 2a) for this experiment. The recorded total ion current decreases below that of the mobile phase within the retention time frame (11-14 min) where high mass oligomers are known to elute, according to the GPC-MALDI MS data (Figure 3e-i). This ion current suppression is not believed to be an instrumental anomaly as it has been repeatedly observed in iterated OH-PDMS sample runs. The ion suppression observed indicates a difference in ion desorption efficiency of the high mass relative to the low mass oligomers (14-16 min) for which a positive ion current relative to baseline is observed. Several experimental factors including added co-solvents and charge agent, drying gas rate, needle position, and all applied voltages within the ESI source need to be considered for detection of the high mass oligomers within the MWD. 
Table 2. Total ion intensity of three poly (dimethyl siloxane) samples obtained from on-line GPC-ESI MS experiments

\begin{tabular}{|c|c|c|c|}
\hline Samples & & HR_PDMS & МF_PDM \\
\hline $\begin{array}{l}\text { Calculated relative total } \\
\text { ion intensity compared } \\
\text { to OH-PDMS }\end{array}$ & $\begin{array}{l}1100 \mathrm{Da} \\
\text { nominal } \\
\text { mass }\end{array}$ & $\begin{array}{l}1700 \mathrm{Da} \\
\text { nominal } \\
\text { mass }\end{array}$ & $\begin{array}{l}1200 \mathrm{Da} \\
\text { nominal } \\
\text { mass }\end{array}$ \\
\hline Measured from TIC & 100 & 76.4 & 69.5 \\
\hline $\begin{array}{l}\text { From summed MS } \\
\text { spectra }\end{array}$ & 100 & 46.7 & 30.1 \\
\hline
\end{tabular}

Although we admit that some optimal combinations of experimental parameters may exist, we were not able to achieve such conditions to remedy the ion suppression that occurred with the high mass oligomers. We propose two possible explanations for this ion suppression phenomenon. First, this may be caused by differences in solubility between high and low mass polysiloxane polymers within desolvating droplets. In general, solubility of a polymer in solution decreases with increasing molecular mass of the polymer [51]. During the rapid desolvation process, the concentration of the polymer in ESI droplets increases dramatically. Perhaps the high mass polymers precipitate out from the partially desolvated droplet, thus precluding transfer to the gas phase. In contrast, the relatively low mass polysiloxane molecule has higher solubility in the desolvating droplet such that it undergoes transfer into the gas phase.

Secondly, it is known that the surface tension of a polymer solution is proportional to the molecular masses of the polymer [52]. We propose that surface tension in the electrospray droplets may play an important role in this observed mass bias. This aspect was discussed previously by Maziarz et al. [53] for ESI-MS analysis of polymer blends. In the current study the mass bias is observed purely as a function of polymer molecular weight eluting from the GPC columns. The higher mass eluting polymers result in a relatively high surface tension that may suppress ESI droplet formation. As the molecular mass of the eluting polymer decreases the surface tension of the ESI droplet decreases such that transfer of the PDMS into the gas phase becomes more favorable. We concede that these two proposals may only partially explain the observed phenomenon. In fact, recent mechanistic studies by Zhou and Cook [54] on the dynamics of the ESI desolvation process may provide further understanding of the underlying principles that cause the ion suppression observed in this study.

Interestingly, we observed differences in ion suppression between the PDMS samples that differed in end group chemistry under constant experimental conditions. Table 2 summarizes the relative signal intensities determined from the TIC and summed mass spectra for each of the three PDMS samples evaluated under constant experimental conditions. This data may suggest that ion desorption efficiency in GPC-ESI-TOF MS experiments for PDMS samples is somewhat dependent

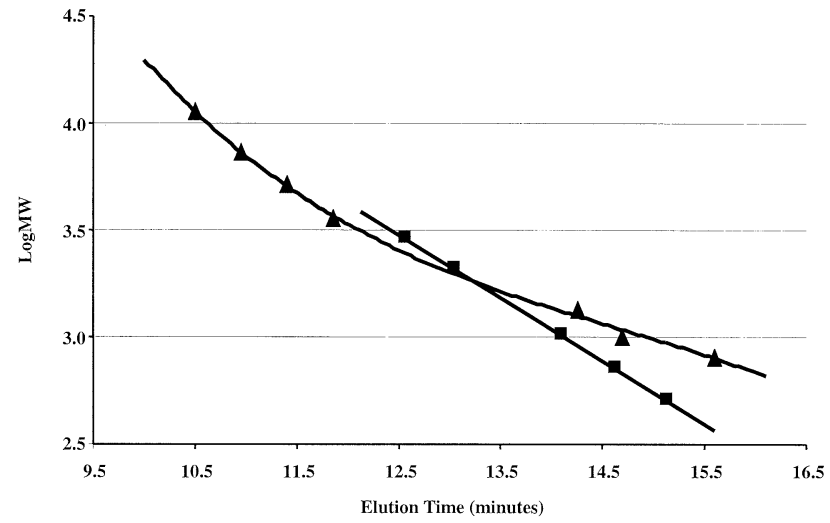

Figure 4. Calibration curves generated from automated GPCMALDI-TOF MS (filled triangle) and on-line GPC-ESI-TOF MS (filled square).

on the polarity of the end group chemistry [53]. A more exhaustive study to evaluate this notion is the topic of future research. Comparatively these same PDMS samples analyzed by GPC-MALDI-TOF MS did not show any difference in signal intensity.

\section{Recalibration of GPC Using Mass Data}

The GPC calibration curve was regenerated from mass values of selected GPC fractions from both hyphenated techniques. Several research groups have also used this method to obtain absolute mass values [10, 11, 39, 40, 42]. The regenerated calibration curves from the two experiments are illustrated in Figure 4. Linear correlation $\left(R^{2}=0.9997\right)$ is obtained in the low mass region by GPC-ESI-TOF MS. It should be noted that there was no data from the high-mass fractions $(>4000 \mathrm{Da})$ available for recalibration. Linear correlation was observed in the higher mass region for GPC-MALDI-TOF MS, however over the broad molecular weight range a third-order polynomial curve fit $\left(R^{2}=0.9981\right)$ was used to construct the calibration curve. The non-linear behavior can be explained by the deviation between hydrodynamic volume and molecular weight over a broad molecular weight range in the GPC size exclusion process. The $M_{n}$ and $\mathrm{M}_{\mathrm{z}}$ values calculated from the recalibrated curve from GPC-ESI-TOF MS experiment (Figure 4) were determined to be approximately $19 \%$ lower than that obtained from traditional GPC. The $M_{n}$ and $M_{z}$ values calculated from the recalibrated method in the GPCMALDI-TOF MS experiment were determined to be 33.5 and $49.1 \%$ higher than that from traditional GPC, respectively. The differences of PD calculated by GPCMALDI and GPC-ESI-TOF MS from that of traditional GPC were determined to be 5.71 and $3.57 \%$, respectively (Table 1).

These results demonstrate that GPC-MALDI-TOF MS can be a useful internal calibration device for a GPC column set over a broad molecular weight range. GPCESI-TOF MS covers only a very narrow portion of the MWD, however it is ideal for calibrating the lowest 
mass portion of the MWD that is sometimes not obtained by MALDI-TOF MS analysis due to chemical noise associated with matrix peaks.

\section{Conclusions}

Both on-line GPC-ESI and automatic GPC-MALDI-TOF MS can be very useful for characterization of polymer samples. GPC-MALDI-TOF MS experiments were performed in a completely automated but off-line manner and provided detailed information on the high-mass polymers within MWD of the PDMS samples evaluated here. However, the information of the polymers on the lower mass ( $<550 \mathrm{Da})$ is completely masked due to the matrix interference. By contrast, GPC-ESI-TOF MS experiments were performed in a true on-line manner. Superior chromatographic resolution was achieved in the low molecular weight range based on the observation of truly mono-disperse PDMS oligomers. The analysis of the higher mass fractions $(>4000 \mathrm{Da})$ was somewhat unsatisfactory due to ion suppression. From this work we anticipate that on-line GPC-ESI and automated GPC-MALDI-TOF MS have potential to be used as high-throughput characterization methods for PDMS materials.

\section{Acknowledgments}

The authors thank Bausch and Lomb, Inc. for its support of this research and for granting the permission of the publication.

\section{References}

1. Yoshida, S.; Yamamoto, S.; Takamatsu, T. Detailed Structural Characterization of Modified Silicone Co-Polymers by MatrixAssisted LaserDesorption/Ionization Time-of-flightMassSpectrometry. Rapid Commun. Mass Spectrom. 1998, 12, 535-544.

2. Wallace, W. E.; Guttman, C. M.; Antonucci, J. M. Molecular Structure of Silsesquioxanes Determined by Matrix-Assisted Laser Desorption/Ionization Time-of-Flight Mass Spectrometry. J. Am. Soc. Mass Spectrom. 1999, 10, 224-230.

3. Maziarz, E. P.; Baker, G. A.; Wood, T. D. Capitalizing on the High Mass Accuracy of Electrospray Ionization Fourier Transform Mass Spectrometry for Synthetic Polymer Characterization: A Detailed Investigation of Poly(dimethylsiloxane). Macromolecules 1999, 32, 4411-4418.

4. Wallace, W. E.; Guttman, C. M.; Antonucci, J. M. Polymeric Silsesquioxanes: Degree of Intramolecular Condensation Measured by Mass Spectrometry. Polymer 2000, 41, 2219-2226.

5. Liu, X. M.; Maziarz, E. P.; Price, F.; Heiler, D. J.; Grobe, G. L. Characterization of Silicone Rubber Extracts Using Gel Permeation Chromatography, Matrix-Assisted Laser Desorption/ Ionization Mass Spectrometry, Electrospray Ionization Mass Spectrometry, Fourier Transform Infrared Spectroscopy, and Gas Chromatography/Mass Spectrometry. Eur. J. Mass Spectrom. 2001, 7, 473-480.

6. Maziarz, E. P.; Liu, X. M.; Quinn, E. T.; Lai, Y.-C.; Ammon, D. M.; Grobe, G. L. Detailed Analysis of $\alpha, \omega$-bis(4-hydroxylbutyl) Poly(dimethylsiloxane) Using GPC-MALDI TOF Mass Spectrometry. J. Am. Soc. Mass Spectrom. 2002, 13, 170-176.

7. Hunt, S.; Cash, G.; Liu, H.; George, G.; Birtwistle, D. Spectroscopic Characterization of Low Molecular Weight Fluids from Silicone Elastomers. J. Macromol. Sci. A 2002, A39, 1007-1024.
8. Yan, W.; Gardella, J. A.; Wood, T. D. Quantitative Analysis of Technical Polymer Mixtures by Matrix Assisted Laser Desorption/Ionization Time of Flight Mass Spectrometry. J. Am. Soc. Mass Spectrom. 2002, 13, 914-920.

9. Yan, W.; Ammon, D. M.; Gardella, J. A.; Maziarz, E. P.; Hawkridge, A. M.; Grobe, G. L.; Wood, T. D. Quantitative Mass Spectrometry of Technical Polymers: A Comparison of Several Ionization Methods. Eur. Mass Spectrom. 1998, 4, 467-474.

10. Montaudo, M. S.; Puglisi, C.; Samperi, F.; Montaudo, G. Application of Size Exclusion Chromatography Matrix-Assisted Laser Desorption/Ionization Time-of-Flight to the Determination of Molecular Masses in Polydisperse Polymers. Rapid Commun. Mass Spectrom. 1998, 12, 519-528.

11. Montaudo, G.; Montaudo, M. S.; Puglisi, C.; Samperi, F. Molecular-Weight Distribution of Poly(dimethylsiloxane) by Combining Matrix-Assisted Laser-Desorption Ionization Time-of-Flight Mass Spectrometry with Gel-Permeation Chromatography Fractionation. Rapid Commun. Mass Spectrom. 1995, 12, 1158-1163.

12. Hanton, S. D. Mass Spectrometry of Polymers and Polymer Surfaces. Chem. Rev. 2001, 101, 527-569.

13. Jackson, C. A.; Simonsick, W. J. Application of Mass Spectrometry to the Characterization of Polymers. Curr. Opin. Solid State Mat. Sci. 1997, 2, 661-667.

14. Montaudo, G. Mass Spectrometry of Synthetic PolymersMere Advances or Revolution. Trip 1996, 4, 81-86.

15. Scrivens, J. H.; Jackson, A. T. Characterization of Synthetic Polymer Systems. Int. J. Mass Spectrom. 2000, 200, 261-276.

16. Montaudo, G.; Montaudo, M. S.; Puglisi, C.; Samperi, F. Characterization of Polymers by Matrix-Assisted Laser Desorption Ionization-Time of Flight Mass Spectrometry. End Group Determination and Molecular Weight Estimates in Poly(ethylene glycols). Macromolecules 1995, 28, 4562-4569.

17. Schriemer, D. C.; Li, L. Detection of High Molecular Weight Narrow Polydisperse Polymers up to 1.5 Million Da by MALDI Mass Spectrometry. Anal. Chem. 1996, 68, 2721-2725.

18. Yalcin, T.; Schriemer, D. C.; Li, L. Matrix-Assisted Laser Desorption Ionization Time-of-Flight Mass Spectrometry for the Analysis of Polydienes. J. Am. Soc. Mass Spectrom. 1997, 8, $1220-1229$.

19. Guttman, C. M.; Blair, W. R.; Danis, P. O. Mass Spectroscopy and SEC of SRM 1487, a Low Molecular Weight Poly(methyl methacrylate) Standard. J. Polym. Sci. Part B-Polym. Phys. 1997, 35, 2409-2419.

20. Guttman, C. M.; Wetzel, S. J.; Blair, W. R.; Fanconi, B. M.; Girard, R. J. G.; Wallace, W. E.; VanderHart, D. L. NISTSponsored Interlaboratory Comparison of Polystyrene Molecular Mass Distribution Obtained by Matrix-Assisted Laser Desorption/Ionization Time-of-Flight Mass Spectrometry: Statistical Analysis. Anal. Chem. 2001, 73, 1252-1262.

21. Axelsson, J.; Scrivener, E.; Haddleton, D. M.; Derrick, P. J. Mass Discrimination Effects in an Ion Detector and Other Causes for Shifts in Polymer Mass Distributions Measured by Matrix-Assisted Laser Desorption/Ionization Time-of-Flight Mass Spectrometry. Macromolecules 1996, 29(27), 8875-8882.

22. Martin, K.; Spickermann, J.; Rader, H. J.; Mullen, K. Why Does Matrix-Assisted Laser Desorption/Ionization Time-of-Flight Mass Spectrometry Give Incorrect Results for Broad Polymer Distributions. Rapid Commun. Mass Spectrom. 1996, 10, 14711474.

23. Hunt, S. M.; Sheil, M. M.; Derrick, P. J. Comparison of Electrospray Ionization Mass Spectrometry with Matrix-Assisted Laser Desorption Ionization Mass Spectrometry and Size Exclusion Chromatography for the Characterization of Polyester Resins. Eur. Mass Spectrom. 1998, 4, 475-486. 
24. Vitalini, D.; Mineo, P.; Scamporrino, E. Effect of Combined Changes in Delayed Extraction Time and Potential Gradient on the Mass Resolution and Ion Discrimination in the Analysis of Polydisperse Polymers and Polymer Blends by Delayed Extraction Matrix-Assisted Laser Desorption/Ionization Time-of-Flight Mass Spectrometry. Rapid Commun. Mass Spectrom. 1999, 13, 2511-2517.

25. Schriemer, D. C.; Li, L. A. Mass Discrimination in the Analysis of Polydisperse Polymers by MALDI Time-of-Flight Mass Spectrometry. 1. Sample Preparation and Desorption/Ionization Issues. Anal. Chem. 1997, 69, 4169-4175.

26. Schriemer, D. C.; Li, L. A. Mass Discrimination in the Analysis of Polydisperse Polymers by MALDI Time-of-Flight Mass Spectrometry. 2. Instrumental Issues. Anal. Chem. 1997, 69, $4176-4183$.

27. Zhu, H.; Yalcin, T.; Li, L. Analysis of the Accuracy of Determining Average Molecular Weights of Narrow Polydispersity Polymers by Matrix-Assisted Laser Desorption Ionization Time-of-Flight Mass Spectrometry. J. Am. Soc. Mass Spectrom. 1998, 9, 275-281.

28. Yalcin, T.; Dai, Y.; Li, L. Matrix-Assisted Laser Desorption/ Ionization Time-of-Flight Mass Spectrometry for Polymer Analysis: Solvent Effect in Sample Preparation. J. Am. Soc. Mass Spectrom. 1998, 9, 1303-1310.

29. Yau, W. W.; Kirkland, J. J.; Bly, D. D. Modern Size-Exclusion Liquid Chromatography. Wiley-Interscience: New York, 1979, Chap. I.

30. Balke, S. T. In Modern Methods of Polymer Characterization; Barth, H. G.; Mays, J. W., Eds.; John Wiley and Sons: New York, 1991, Chap. I.

31. Shi, S. D. H.; Hendrickson, C. L.; Marshall, A. G.; Simonsick, W. J.; Aaserud, D. J. Identification, Composition, and Asymmetric Formation Mechanism of Glycidyl Methacrylate Butyl Methacrylate Copolymers up to 7000 Da from Electrospray Ionization Ultrahigh Resolution Fourier Transform Ion Cyclotron Resonance Mass Spectrometry. Anal. Chem. 1998, 70, 3220-3226.

32. Lou, X. W.; van Dongen, J. L. J.; Meijer, E. W. Off-Line Size-Exclusion Chromatographic Fractionation-Matrix-Assisted Laser Desorption Ionization Time-of-Flight Mass Spectrometry for Polymer Characterization-Theoretical and Experimental Study. J. Chromatogr. A 2000, 896, 19-30.

33. Montaudo, G.; Garozzo, D.; Montaudo, M. S.; Puglisi, C.; Samperi, F. Molecular and Structural Characterization of Polydisperse Polymers and Copolymers by Combining MALDI-TOF Mass Spectrometry with GPC Fractionation. Macromolecules 1995, 28, 7983-7989.

34. Montaudo, G.; Montaudo, M. S.; Puglisi, C.; Samperi, F. Characterization of Polymers by Matrix-Assisted Laser-Desorption Ionization Time-of-Flight Mass Spectrometry-Molecular-Weight Estimates in Samples of Varying Polydispersity. Rapid Commun. Mass Spectrom. 1995, 9, 453460.

35. Montaudo, M. S.; Puglisi, C.; Samperi, F.; Montaudo, G. Molar Mass Distributions and Hydrodynamic Interactions in Random Copolyesters Investigated by Size Exclusion Chromatography Matrix-Assisted Laser Desorption Ionization. Macromolecules 1998, 31, 3839-3845.

36. Montaudo, M. S.; Montaudo, G. Bivariate Distribution in PMMA/PBA Co-Polymers by Combined SEC/NMR and SEC/MALDI Measurements. Macromolecules 1999, 32, 70157022

37. Prokai, L.; Simonsick, W. J. Electrospray-Ionization Mass Spectrometry Coupled with Size-Exclusion Chromatography. Rapid Commun. Mass Spectrom. 1993, 7, 853-856.
38. Simonsick, W. J.; Prokai, L. Size Exclusion Chromatography with Electrospray Mass-Spectrometric Detection. Adv. Chem. Ser. 1995, 247, 41-56.

39. Aaserud, D. J.; Prokai, L.; Simonsick, W. J. Gel Permeation Chromatography Coupled to Fourier Transform Mass Spectrometry for Polymer Characterization. Anal. Chem. 1999, 71, 4793-4799.

40. Prokai, L.; Aaserud, D. J.; Simonsick, W. J. Microcolumn Size-Exclusion Chromatography Coupled with Electrospray Ionization Mass Spectrometry. J. Chromatogr. A. 1999, 835, 121-126.

41. Fei, X.; Murray, K. K. On-line Coupling of Gel-Permeation Chromatography with MALDI Mass Spectrometry. Anal. Chem. 1996, 68, 3555-3560.

42. Hanton, S. D.; Liu, X. M. GPC Separation for MALDI Analysis. Anal. Chem. 2000, 72, 4550-4554.

43. Maziarz, E. P.; Liu, X. M. A Modified Thermal Deposition Unit for GPC MALDI-TOF MS and ESI-TOF MS Analysis. Eur. J. Mass Spectrom. 2002, 8, 397-401.

44. Kassis, C. E.; DeSimone, J. M.; Linton, R. W.; Remsen, E. E.; Lange, G. W.; Friedman, R. M. A Direct Deposition Method for Coupling Matrix-Assisted Laser Desorption/Ionization Mass Spectrometry with Gel Permeation Chromatography for Polymer Characterization. Rapid Commun. Mass Spectrom. 1997, 11, 1134-1138.

45. Yun, H.; Olesik, S. V.; Marti, E. H. Polymer Characterization Using Packed Capillary Size Exclusion and Critical Adsorption Chromatography Combined with MALDI-TOF Mass Spectrometry. J. Microcol. Sep. 1999, 11, 53-61.

46. Lou, X. W.; van Dongen, J. L. J. Direct Sample Fraction Deposition Using Electrospray in Narrow-Bore Size-Exclusion Chromatography/Matrix-Assisted Laser Desorption/Ionization Time-of-Flight Mass Spectrometry for Polymer Characterization. J. Mass Spectrom. 2000, 35, 1308-1312.

47. Nielen, M. W. F. Polymer Analysis by Micro-Scale Size Exclusion Chromatography MALDI Time-of-Flight Mass Spectrometry with a Robotic Interface. Anal. Chem. 1998, 70, 15631568.

48. Dwyer, J.; Botten, D. A Novel Sample Preparation Device For MALDI-MS. Am. Lab. 1996, 28, 51-54.

49. Lai, Y. C.; Ozark, R.; Quinn, E. T. Synthesis and Characterization of $\alpha, \omega$-bis(4-hydroxylbutyl) Polydimethylsiloxanes. J. Polym. Sci. Chem. Part A 1995, 33, 1773-1782.

50. Maziarz, E. P.; Liu, X. M.; Ammon, D.; Grobe, G. L.; Bonafini, J. Analysis of Complex Polymer Mixtures by GPC-MALDI TOF MS: Instrumental Modifications and Comparison to Direct Analysis. Proceedings of the 49th ASMS Conference on Mass Spectrometry and Allied Topics; Chicago, IL, 2001; CD Edition.

51. Carraher, C. E., Jr. Seymour/Carraher's Polymer Chemistry: An Introduction. Marcel Dekker: New York, 1996; 4th ed.; pp $59-67$.

52. Wu, S. Surface and Interfacial Tensions of Polymers, Oligomers, Plasticizers, and Organic Pigments. Brandrup, J.; Immergut, E. H.; Grulles, E. A., Eds.; Wiley: New York, 1999; 4th ed.; pp 521-541. In: Polymer Handbook, Vol. VI.

53. Maziarz, E. P.; Baker, G. A.; Mure, J. V.; Wood, T. D. A Comparison of Electrospray Versus Nanoelectrospray Ionization Fourier Transform Mass Spectrometry for the Analysis of Synthetic Poly(dimethylsiloxane)/Poly(ethylene glycol) Oligomer Blends. Int. J. Mass Spectrom. 2000, 202, 241-250.

54. Zhou, S.; Cook, K. D. A Mechanistic Study of Electrospray Mass Spectrometry: Charge Gradients Within Electrospray Droplets and Their Influence on Ion Response. J. Am. Soc. Mass Spectrom. 2000, 12, 206-214. 